\title{
Estudio electrofisiológico y electroacústico de un desorden del espectro de neuropatía auditiva: Reporte de un caso en recién nacido de alto riesgo
}

\section{Electrophysiology and electroacoustic study of spectrum disorder of auditory neuropathy: Report of a case in high-risk newborn}

\author{
Oscar Cañete $\mathbf{S}^{1}$, Fabiola Aránguiz $\mathrm{L}^{2}$.
}

\begin{abstract}
RESUMEN
El desorden del espectro de neuropatía auditiva (DENA) describe una condición en la cual se observa o se ha observado emisiones otoacústicas (EOA) junto con potenciales evocados auditivos de tronco (PEAT) anormales o ausentes. Su identificación se basa en la presencia de potencial microfónico coclear (PMIC) junto con un PEAT anormal o ausente y EOA presentes o antecedentes de su presencia. Los umbrales tonales pueden encontrarse entre los rangos de normalidad hasta pérdidas auditivas profundas. El reconocimiento del habla generalmente se encuentra afectado particularmente en presencia de ruido de fondo, sin embargo en algunos individuos se mantiene conservado (sin ruido).

Se presenta caso clínico de un recién nacido de sexo femenino de pretérmino de 29 semanas y 810 grs (GIII), apgar 7/8, presenta displasia broncopulmonar, sospecha de infección connatal tratada, hiperbilirrubinemia ( $\max 11 \mathrm{mg} / \mathrm{dl}$ ) tratada con fototerapia. Es derivada al Servicio de Otorrinolaringología para su evaluación auditiva. La evaluación auditiva realizada corresponde a potenciales evocados auditivos de tronco (PEAT), impedanciometria, audiometría de campo de respuesta reflejas y emisiones otoacústicas. Los resultados obtenidos concuerdan con lo descrito en la literatura para un DENA.

Palabras claves: Neuropatía auditiva, Desorden del espectro de neuropatía auditiva, Hipoacusia neural, Hipoacusia en neonatos de riesgo, Potenciales evocados auditivos de tronco cerebral.
\end{abstract}

\section{ABSTRACT}

Auditory neuropathy spectrum disorder (ANSD) describes a condition in which it is possible to find otoacoustic emissions (OAE) in presence of abnormal or absent auditory brainstem evoked potentials (ABR). The pure tone thresholds may show a

\footnotetext{
${ }^{1}$ Tecnólogo Médico, Unidad de Audiología Clínica, Servicio de Otorrinolaringología, Hospital Padre Hurtado.

${ }^{2}$ Tecnólogo Médico, División Investigación y Docencia, Signalmed Ltda.
} 
spectrum from normal to profound hearing loss. Speech recognition is often affected in presence of background noise, but in some individuals remains intact.

We report a case of a pre term newborn (female) of 29 weeks and 810 grams, Apgar $7 / 8$, bronchopulmonary dysplasia, suspected connatal infection, and hyperbilirubinemia (max $11 \mathrm{mg} / \mathrm{dl}$ ). She was referred to Hospital Padre Hurtado for hearing assessment. The hearing test performed were an ABR, tympanometry \& acoustic reflex, visual reinforcement audiometry and otoacoustic emissions. The results are consistent with those described in the literature for ANSD.

Key words: Auditory neuropathy, Auditory neuropathy spectrum disorder, auditory evoked potentials, cochlear microphonic.

\section{INTRODUCCIÓN}

El desorden del espectro de neuropatía auditiva (DENA) describe una condición en la cual se observa 0 se ha observado emisiones otoacústicas (EOA) junto con potenciales evocados auditivos de tronco (PEAT) anormales o ausentes. Su identificación se basa en la presencia de potencial microfónico coclear (PMIC) junto con un PEAT anormal 0 ausente y EOA presentes 0 antecedentes de su presencia. Los umbrales tonales pueden encontrarse entre los rangos de normalidad hasta pérdidas auditivas profundas. El reconocimiento del habla generalmente se encuentra afectado particularmente en presencia de ruido de fondo, sin embargo en algunos individuos se mantiene conservado (sin ruido). Así mismo se han reportado casos en donde hay hipoacusia fluctuante, algunos de estos casos asociados a cambios patológicos de temperatura corporal'.

La prevalencia de un DENA se estima entre rangos que van desde $0,23 \%$ a $0,94 \%$ en infantes con factores de riesgo de presentar una pérdida auditiva, se ha reportado una prevalencia de incluso de $1,96 \%$ en individuos egresados de una unidad de cuidados intensivos neonatal. Entre los niños diagnosticados con una pérdida auditiva permanente la prevalencia de este desorden puede alcanzar el $7 \% 0$ incluso el $11 \%{ }^{2}$.

Características de un DENA han sido reportadas en pacientes con antecedentes de prematuridad, hiperbilirrubinemia, asfixia perinatal, la ventilación mecánica, varios procesos infecciosos. También en anomalías genéticas, la cuales incluyen alteraciones en genes como OTOF, PMP22, MPZ, y NDRGI ${ }^{3}$.

El uso de los audífonos ayudaría al paciente al acceso de los elementos del habla y/o mejorando la sincronización neural estimulando todas las neuronas disponibles. Así mismo el uso de un implante coclear (IC) podría ser un tratamiento para estos pacientes, esto dirigido a restaurar el procesamiento de la información auditiva a través de la estimulación eléctrica. En la actualidad existe evidencia sobre la ventaja de la implantación en pacientes con DENA. Sin embargo el tratamiento debe ser individual para cada individuo debido a la heterogeneidad de presentación de un DENA².

El objetivo de este trabajo es exponer un caso clínico en donde los hallazgos audiológicos observados son compatibles con un DENA.

\section{CASO CLÍNICO}

Recién nacido de sexo femenino de pretérmino de 29 semanas y 810 grs (GIII), apgar 7/8, presenta displasia broncopulmonar, sospecha de infección connatal tratada, hiperbilirrubinemia $(\max 11 \mathrm{mg} / \mathrm{dl})$ tratada con fototerapia. Es derivada al Servicio de Otorrinolaringología para su evaluación auditiva. Esta se inicia con una etapa de pesquisa y diagnóstico. Se realizan potenciales evocados automatizados de tronco (aPEAT) que refieren en forma bilateral en sus 2 etapas, según programa se deriva a PEAT clínico (estímulo click). En la evaluación clínica a 90 $\mathrm{dBnHL}$ no se observa respuesta en oído derecho (OD) y en oído izquierdo (OI) se observa umbral de onda $\mathrm{V}$ a $80 \mathrm{dBnHL}$ de mala morfología y replicabilidad, la primera evaluación se realiza al mes de edad corregida y la segunda a los 3 meses, en ambas se obtiene similar resultado (Tabla 1).

A los 7 meses de edad corregida se realiza audiometría a campo libre de respuestas reflejas, su resultado sugiere una pérdida auditiva profunda (Figura 1). 
Tabla 1. Resumen de resultados evaluación auditiva

\begin{tabular}{|c|c|c|c|}
\hline & & OD & 이 \\
\hline Pesquisa & aPEAT (Agos-2008) & Refiere & Refiere \\
\hline \multirow[t]{3}{*}{ Diagnóstico } & $\begin{array}{l}\text { PEAT Clínico } 1 \text { (Sept-2008) } \\
\text { Estímulo click }\end{array}$ & Sin respuesta observable & $\begin{array}{l}\text { Se observa onda } V \text { a } 80 \mathrm{dBnHL} \\
\text { de mala morfología y no replicable }\end{array}$ \\
\hline & $\begin{array}{l}\text { PEAT Clínico } 2 \text { (0ct-2008) } \\
\text { Estímulo click }\end{array}$ & Sin respuesta & $\begin{array}{l}\text { Se observa onda } V \text { a } 80 \mathrm{dBnHL} \\
\text { de mala morfología y no replicable }\end{array}$ \\
\hline & Audio campo libre (Dic-2008) & \multicolumn{2}{|c|}{$\begin{array}{l}\text { Hipoacusia profunda solo con restos auditivos en } 250 \text { y } 500 \mathrm{~Hz} \\
\text { SDT a la voz } 55 \mathrm{~dB} \mathrm{HL}\end{array}$} \\
\hline \multirow[t]{6}{*}{$\begin{array}{l}\text { Control y } \\
\text { seguimiento }\end{array}$} & $\begin{array}{l}\text { PEAT Clínico } 3 \text { (Abr-2010) } \\
\text { Estímulo click } \\
\text { Estímulo Burst }\end{array}$ & $\begin{array}{l}\text { Se observa onda V a } 99 \mathrm{dBnHL} \\
\text { de mala morfología } \\
\text { y no replicable/PMC }\end{array}$ & $\begin{array}{l}\text { Se observa onda } V \text { a } 99 \mathrm{dBnHL} \\
\text { de mala morfología } \\
\text { y no replicable/PMC }\end{array}$ \\
\hline & $\begin{array}{l}\text { - Emisiones otoacústicas PD } \\
\text { - Timpanograma (226 Hz) }\end{array}$ & $\begin{array}{l}\text { Presentes } \\
\text { Curva "C" }\end{array}$ & $\begin{array}{l}\text { Presentes } \\
\text { Curva "C" }\end{array}$ \\
\hline & Audio campo libre (Abr-2010) & \multicolumn{2}{|c|}{$\begin{array}{l}\text { Umbrales entre los } 20 \text { y } 35 \mathrm{dBHL} \text {, rangos de frecuencia de } 250 \text { y } 4000 \mathrm{~Hz} \\
\text { SDT a la voz } 15 \mathrm{~dB} \mathrm{HL}\end{array}$} \\
\hline & PEAT clínico 4 (Abr-2010) & Se observa PMC & Se observa PMC \\
\hline & $\begin{array}{l}\text { - Emisiones otoacústicas PD } \\
\text { - Timpanograma ( } 256 \mathrm{~Hz}) \\
\text { - Reflejo acústico (ipsilateral) }\end{array}$ & $\begin{array}{l}\text { Presentes } \\
\text { Curva "A" } \\
\text { Negativo }\end{array}$ & $\begin{array}{l}\text { Presentes } \\
\text { Curva "A" } \\
\text { Negativo }\end{array}$ \\
\hline & Audio campo libre (Jul-2010) & \multicolumn{2}{|c|}{$\begin{array}{l}\text { Umbrales entre los } 15 \text { y } 25 \mathrm{dBHL} \text {, rangos de frecuencia de } 250 \text { y } 4000 \mathrm{~Hz} \\
\text { SDT a la voz } 10 \mathrm{~dB} H \mathrm{HL}\end{array}$} \\
\hline
\end{tabular}

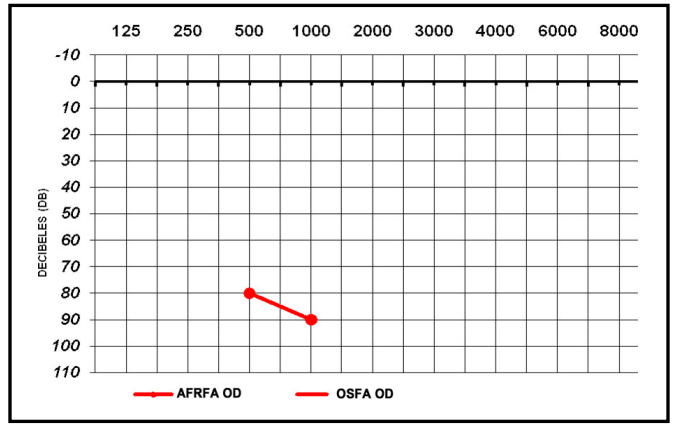

Figura 1. Audiometría a campo libre de respuestas reflejas con refuerzo visual (7 meses).

Considerando los resultados de los exámenes realizados se procede a la confirmación diagnóstica (prematuro GES) y la implementación de audífonos en forma bilateral (audífonos retroauriculares de alta potencia con WDRC y tres canales de programación) más sistema FM (año 2008). La paciente ingresa al programa de habilitación auditiva el cual incluye terapia auditiva verbal, con fecha julio 2010 la evaluación fonoaudiológica concluye que presenta un retraso del lenguaje encontrándose en un desarrollo de 12 a 14 meses aproximadamente. Se comunica con uso de jerga y sintagmas nominales (mamá, papá, guagua). Usa onomatopeya de algunos animales como gato, perro, caballo, oveja y del auto. Utiliza gestos como estrategia de compensación.

A nivel comprensivo responde a su nombre y el "No". Presenta juego simbólico y asocia uso de objetos. No identifica elementos de categorías básicas por su nombre, con apoyo gestual logra identificar unos pocos.

El proceso continúa con la etapa de seguimiento y control. Durante el transcurso del año 2009 su 
asistencia a terapia fue irregular así como el uso constante de los audífonos. Durante el mes de enero de 2010 asiste nuevamente a sesiones de rehabilitación auditiva, debido al tiempo transcurrido desde la implementación con audífonos se realiza evaluación auditiva de control (abril 2010).

A los 2 años (mayo 2010) es evaluada por neurólogo infantil descartándose alguna patología neurológica asociada, se le diagnostica un trastorno conductual por lo que es derivada a terapia ocupacional.

\section{Resultados PEAT}

En la evaluación control de PEAT (estimulo Click). A 90 y $99 \mathrm{~dB} \mathrm{nHL}$ se observa en ambos oídos un trazado con morfología alterada en donde no es posible identificar las ondas I, II, III, IV ni V. (Figuras 2, 3). El registro de su trazado, no es replicable al estimular en dos oportunidades a una misma intensidad ni al comparar los resultados de evaluaciones previas (Tabla 1).

Al estimular utilizando un tono burst de $1.000 \mathrm{~Hz}$ como tono de prueba no se observa respuesta de onda I, II, III, IV ni V a 100 dB n HL (Figuras 4, 5).

Los resultados de todos los PEAT realizados sugieren hipoacusia bilateral severa a profunda para las frecuencias entre 1.000 y $4.000 \mathrm{~Hz}$.

En las evaluaciones se observa la presencia del PMIC en ambos oídos entre los 0,5 a 1,8 mseg con mayor amplitud en oído derecho, así mismo el PMIC se evidencia con mayor claridad a altas intensidades de estimulación (80 y $90 \mathrm{dbB} n \mathrm{HL}$ ) comparado con menores intensidades (70 y $60 \mathrm{~dB}$ $\mathrm{n} \mathrm{HL}$ ) (Figuras 6, 7). La presencia del PMIC se evidencia al comparar la respuesta obtenida al estimular mediante polaridad alternada en donde se utilizan estímulos con polaridad en condensación y en rarefacción, dicha respuesta se separa en la mitad de las promediaciones en dos memorias, en memoria $A$ se almacenan la mitad de las promediaciones, es decir, las respuesta con polaridad condensación y en memoria B se almacenan la otra mitad de promediaciones que corresponden a las respuesta obtenidas con polaridad rarefacción.

\section{Resultados EOAs}

Para el estudio de la función coclear se realizaron EOA producto de distorsión ( $p d$ ) en las frecuencias de 500, 750, 1.000, 1.500, 2.000, 3.000, 4.000,

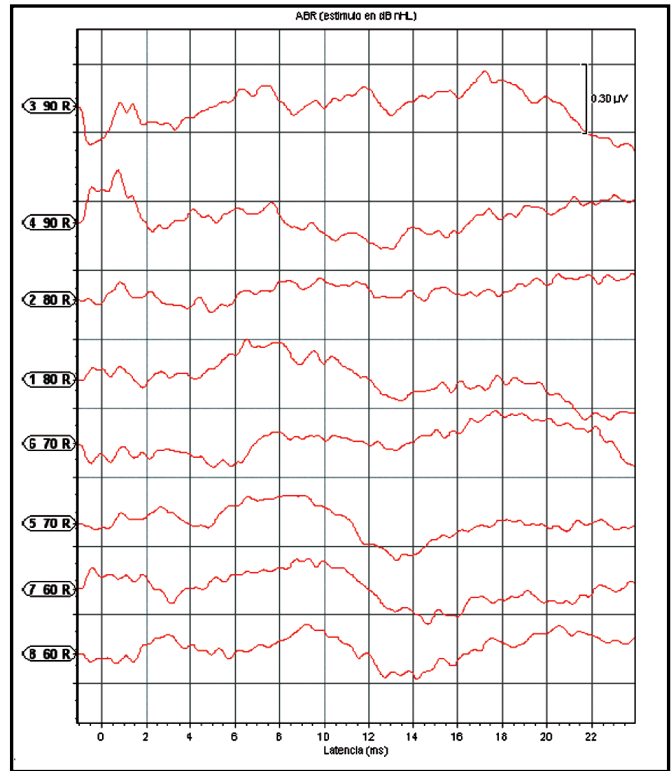

Figura 2. Registro I PEAT de oído derecho, estímulo click (abril 2010).

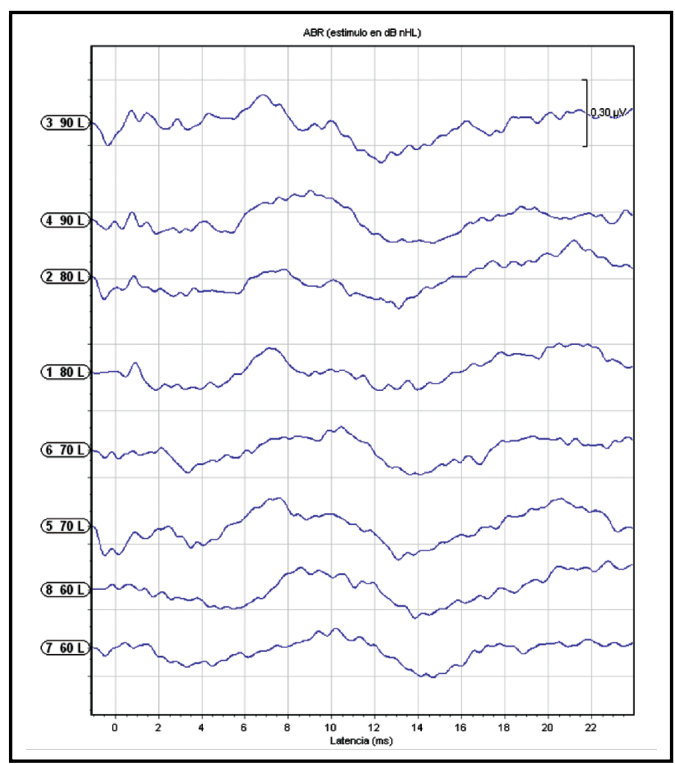

Figura 3. Registro II PEAT de oído izquierdo, estimulo click (abril 2010).

6.000 y $8.000 \mathrm{~Hz}$. Se registran EOApd en ambos oídos de baja amplitud presentado una relación señal ruido $(S / R)>5 d B$. Ver Tabla 1 .

En oído derecho se observan EOApd en las frecuencias de 1.500, 2.000, 4.000, 6.000 y $8.000 \mathrm{y}$ 


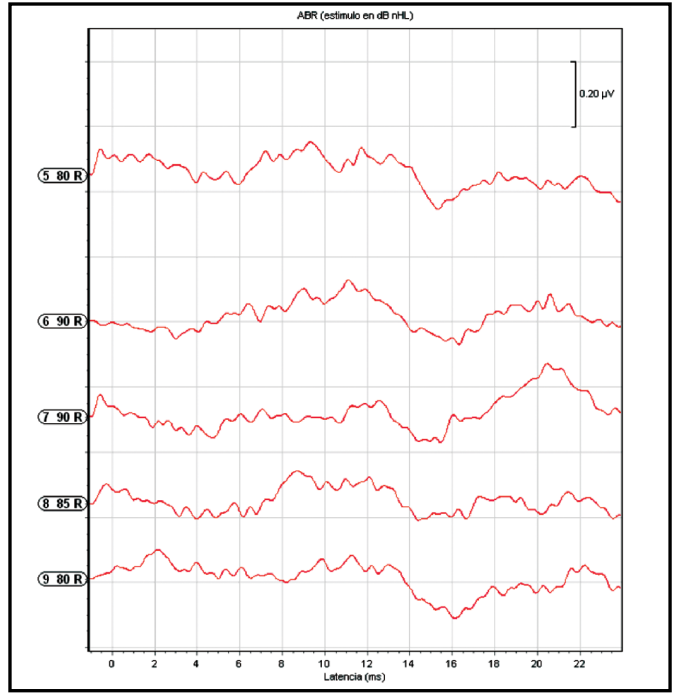

Figura 4. Registro II PEAT de oído derecho, estímulo Burst $1.000 \mathrm{~Hz}$ (abril 2010).

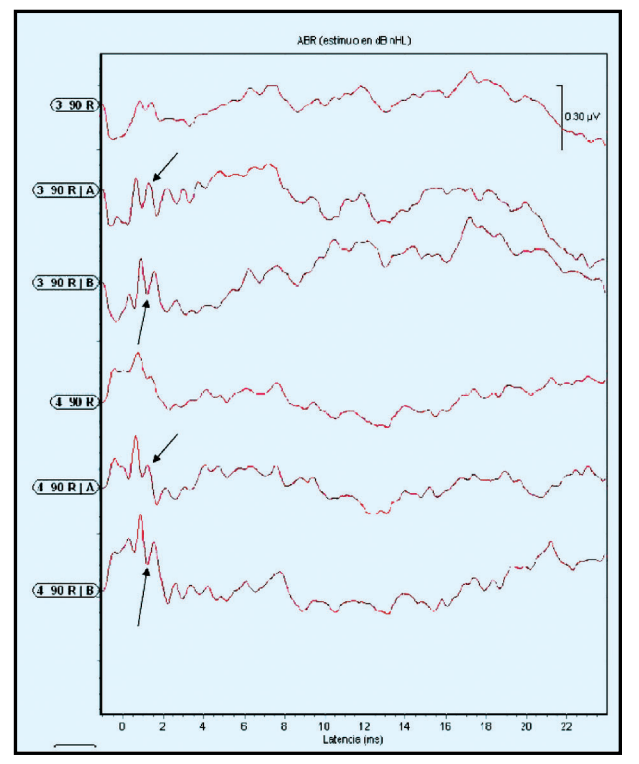

Figura 6. Registro PEAT de oído derecho, estimulo click A-B. A: Polaridad de condensación. B: Polaridad de rarefacción.

en oído izquierdo en las frecuencias de 3.000, 4.000, 6.000 y $8.000 \mathrm{~Hz}$ (Ver Figuras 8, 9). Los resultados de las EOA muestran actividad de las células ciliadas externas de la cóclea en ambos oídos.

\section{Resultado de evaluación electroacústica}

En una primera evaluación se observa timpanograma "C" bilaterales (tono prueba $226 \mathrm{~Hz}$ ) con ausencia de

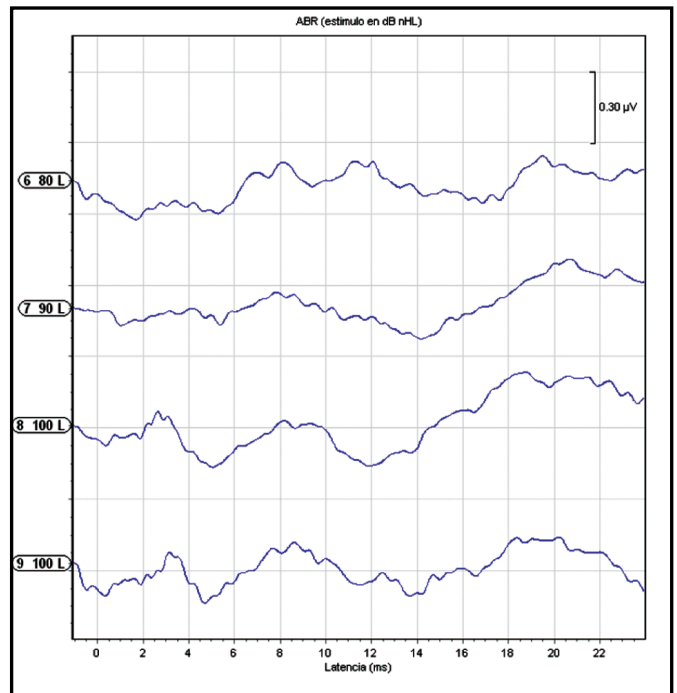

Figura 5. Registro PEAT de oído izquierdo estímulo Burst $1.000 \mathrm{~Hz}$ (Abril 2010).

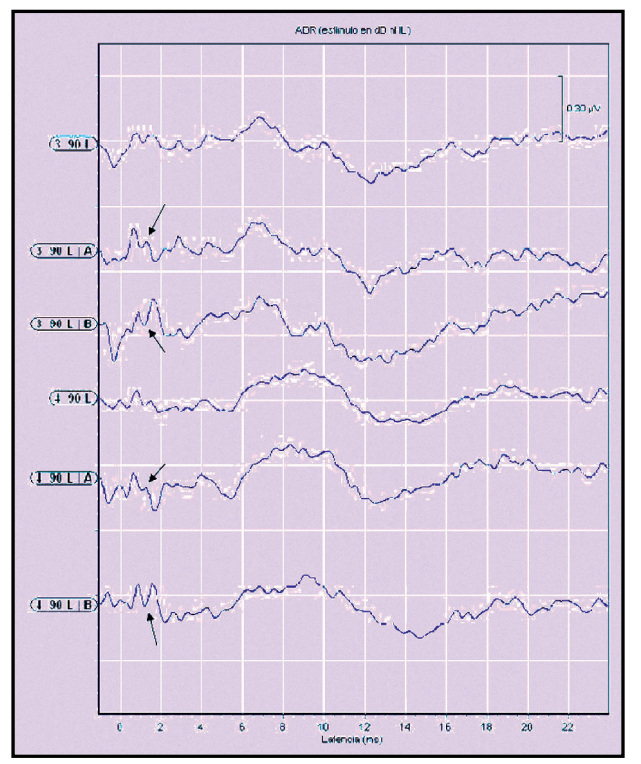

Figura 7. Registro PEAT de oído izquierdo, estímulo click A-B

reflejo acústico (ipsilaterales). Segunda evaluación se observa timpanograma "A" bilaterales (tono prueba $226 \mathrm{~Hz}$ ) con ausencia de reflejo acústico (ipsilaterales).

\section{Resultado evaluación conductual}

Las mediciones conductuales en una primera oportunidad (audiometría de campo con refuerzo vi- 


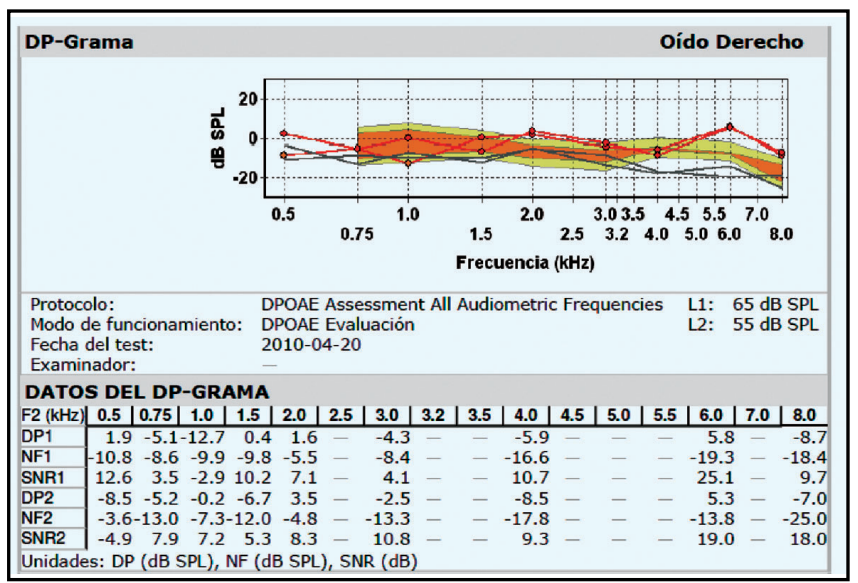

Figura 8. Registro de emisiones otoacústicas PD en oído derecho.

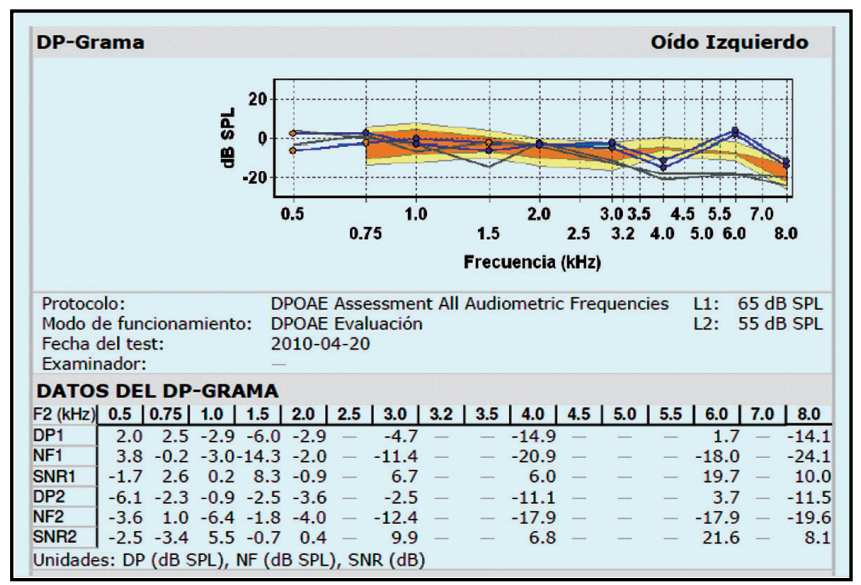

Figura 9. Registro de emisiones otoacústicas PD en oído izquierdo.

sual) muestran respuestas sugerentes de una hipoacusia severa a profunda, en una segunda evaluación de control es sugerente de una pérdida leve. Debido a la edad del paciente no evalúa discriminación auditiva, sin embargo se logra establecer umbral de detección de la voz en ambas sesiones (Ver Figuras 10, 11).

En el presente caso los resultados son sugerentes de una alteración en la vía auditiva compatible con una DENA, demostrado por PEATs alterados junto con la evidencia de actividad coclear dada por la presencia de EOAs, y resultados de la medición conductual sin relación con las mediciones electrofisiológicas y electroacústicas realizadas.

Ante los antecedentes expuestos y la evidencia existente en la literatura se decide suspender el uso continuo de los audífonos con el objetivo de evitar un posible daño de las células ciliadas², sin embargo se continúa con sesiones de estimulación auditiva y controles periódicos de audición conductual.

\section{DISCUSIÓN}

La actividad sincrónica del nervio auditivo en el tronco cerebral puede ser evaluada a través del PEAT. Esta medición refleja la actividad neural sincrónica frente a un estímulo acústico. Una alteración de tal actividad podría afectar la codificación temporal a nivel del tronco, afectando por lo tanto la obtención de las respuestas del PEAT. Los resultados obtenidos concuerdan con lo descrito en la literatura en donde 


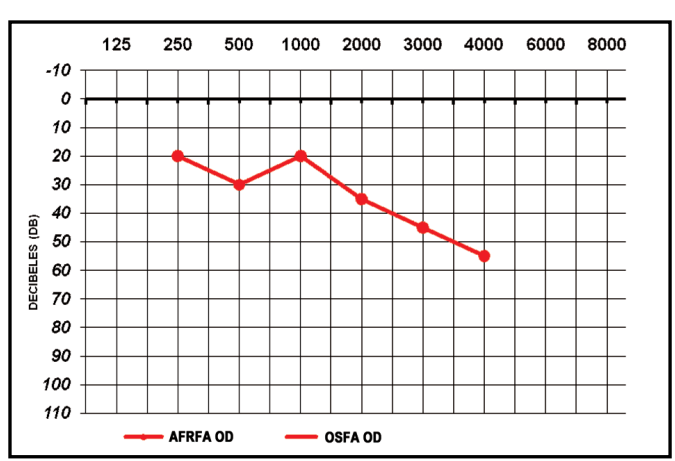

Figura 10. Audiometría a campo libre de respuestas reflejas con refuerzo visual (1 año 11 meses).

se señala una alteración importante de las respuestas del PEAT, es así como se pudo observar respuestas de mala morfología y replicabilidad ${ }^{4}$.

Es así como en algunas series de paciente con un DENA se ha observado la ausencia completa de respuestas en el PEAT de hasta el $70 \%$ de los casos sin importar la intensidad de estimulación. El 19\% muestra sólo la presencia de onda $\mathrm{V}$, presentado peaks mal definidos (mala morfología), latencia alterada y baja amplitud. En el 6\% se pudo observar respuestas anormales pero con presencia de al menos 2 peaks, usualmente onda III y V. De igual modo estas respuestas son de mala morfología y amplitud ${ }^{5}$.

El potencial microfónico coclear (PMIC) refleja la actividad de las células ciliadas por lo que se considera un potencial preneural. Mediante la utilización de diferentes polaridades de estimulación (condensación/rarefacción) es posible observar la presencia del PMIC ya que la polaridad del estímulo determina la polaridad del registro. En pacientes con DENA es posible encontrar el PMIC debido a que éste reflejaría la actividad de las células ciliadas las cuales no estarían afectadas en este desorden ${ }^{6}$.

La presencia 0 antecedentes de actividad coclear dada por la presencia de EOAs es una característica frecuente en los individuos con un DENA, algunos reportes de casos van desde el $75 \%$ a $80 \%$ de presencia en ambos oídos de EOAs ${ }^{1,5}$. Existen algunos autores que señalan la posible disminución o desaparición de EOAs debido a una "sobreamplificación" en presencia de actividad coclear, hecho que aún no está del todo claro. También se ha observado la ausencia de EOAs en individuos con DENA no usuarios de audífonos ${ }^{2,5}$.

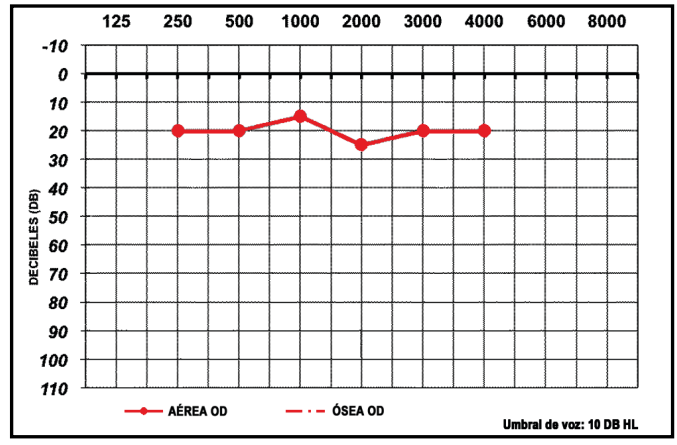

Figura 11. Audiometría a campo libre de respuestas reflejas con refuerzo visual (2 años 2 meses).

En relación a la función del oído medio en estos pacientes, ésta se encuentra conservada, observándose una buena movilidad de la membrana timpánica. En el caso de la presencia de reflejo acústico (RA), en estos pacientes generalmente se encuentra ausente. Berlin $\mathrm{CH}$, Hood y cols (2010), revisaron una serie de 260 pacientes con diagnóstico de DENA encontrándose la ausencia de RA en el $90 \%$ de éstos. Aquellos que presentaban RA presentaban una combinación de presencia (elevados) y ausencia de éstos ${ }^{1}$. En el presente caso se observa la ausencia de RA en ambos oídos (reflejos ipsilaterales).

La evaluación auditiva conductual debe ser incluida como un elemento de estudio de un DENA, ésta nos ayuda a determinar el impacto de tal desorden sobre la sensibilidad auditiva del individuo. Una evaluación mediante una audiometría de refuerzo visual debe ser realizada tan pronto como sea posible, generalmente sobre los 6 meses de edad, esto con el fin de establecer los umbrales auditivos. Frecuentemente un DENA se asocia a una sensibilidad auditiva fluctuante, por lo que esta evaluación debe ser realizada periódicamente de modo de establecer el grado y configuración de la posible pérdida ${ }^{7}$.

Los umbrales auditivos conductuales se observan desde rangos normales hasta pérdidas profundas. Frecuentemente estas respuestas se consideran de pobre confiabilidad, siendo éstas en muchas ocasiones variables de evaluación en evaluación ${ }^{8-10}$.

En la actualidad podemos observar que la incidencia del diagnóstico de un DENA ha aumentado, esto se debería a la implementación de programas de pesquisa precoz de hipoacusia en recién nacidos así como el aumento de la supervi- 
vencia de prematuros incluso de aquellos menores de 30 semanas de gestación.

\section{CONCLUSIÓN}

Es de suma importancia la utilización de las técnicas de evaluación adecuadas en especial en aquellos pacientes con algún factor de riesgo de presentar una hipoacusia. En las evaluaciones de menores con factores de alto riesgo, además de utilizar distintos exámenes complementarios, el criterio de evaluación debe considerar la búsqueda de hallazgos para diferenciar patologías sensoriales de neurales, en estos casos la evidencia del PMIC y presencia 0 antecedentes de actividad coclear.

Es importante una interpretación cuidadosa de los resultados obtenidos en niños que presentan factores de riesgo especialmente al utilizar EOA 0 aPEAT como única herramienta de pesquisa. Al utilizar sólo un método de evaluación auditiva (EOAs 0 aPEAT) en pacientes de alto riesgo se pierde la posibilidad de pesquisar un DENA.

Es necesario el control y seguimiento audiológico periódico de los pacientes diagnosticados con una pérdida auditiva sugerente de DENA. Junto con los métodos más utilizados en la evaluación de recién nacidos o niños pequeños no se debe olvidar la importancia de la evaluación conductual, estudiada mediante una audiometría tonal o audiometría de campo libre según corresponda.

En el caso de un diagnóstico de DENA es necesaria la individualización de cada plan de tratamiento. Debido a lo anterior en estos pacientes el seguimiento es de gran relevancia para determinar el tipo de intervención adecuada cautelando tanto el estado auditivo como su desarrollo de habla y lenguaje.

\section{Agradecimientos}

Dra. Linda J Hood PhD. Department of Hearing and Speech Sciences. Vanderbilt University. Dr James
Hall III PhD. Department of Speech, Language, and Hearing Sciences. University of Florida.

\section{BIBLIOGRAFÍA}

1. Berlin Cl, Hood LJ, Morlet T, Wilensky D, Li L, y coLs. Multi-site diagnosis and management of 260 patients with auditory neuropathy/dyssynchrony (auditory neuropathy spectrum disorder. Int J Audiol 2010; 49(1): 30-43.

2. Vlastarakos PV, Nikolopoulos tP, Tavoulari E, Papacharalambous G, Korres S. Auditory neuropathy: Endocochlear lesion or temporal processing impairment? Implications for diagnosis and management. Int $J$ Pediatr Otorhinolaryngol 2008; 72: 8: 1135-50.

3. Roush P. Auditory neuropathy spectrum disorder: Evaluation and management. Hear $J$ 2008; 61: 11.

4. Zeng F, Oba S, Garde S, Sininger I, Starr A. Temporal and Speech processing deficits in auditory neuropathy. Neuroreport 1999; 10(16): 3429-35.

5. Sininger Y. Starr A. Auditory Neuropathy. Singular Thompson Learing. $1^{\text {a }}$ edición. 2001.

6. Berlin $\mathrm{CH}$, Morlet T, Hood L. Auditory Neuropathy/Dyssynchrony. Its diagnosis and management. Pediatr Clin N Am 2003; 50: 33140.

7. Wolfe J \& Kasulis H. (2008, February 12). Contemporary Audiologic Assessment for Auditory Dys-Synchrony. The ASHA Leader.

8. Rose K, Hood LJ, BerLin CH. An Overview of the Evolution of Auditory Neuropathy-Its Diagnosis and Management. Perspectives on Hearing and Hearing Disorders in Childhood 2002; 12: 3-11.

9. Rance G. Auditory Neuropathy/Dys-synchrony and it's Perceptual Consequences. Trends Amplif 2005; 9(1): 1-43.

10. Rance G, Bee DE, Cone-Wesson B, et al. Clinical findings for a group of infants and young children with auditory neuropathy. Ear Hear 1999; 20: 238-52. 